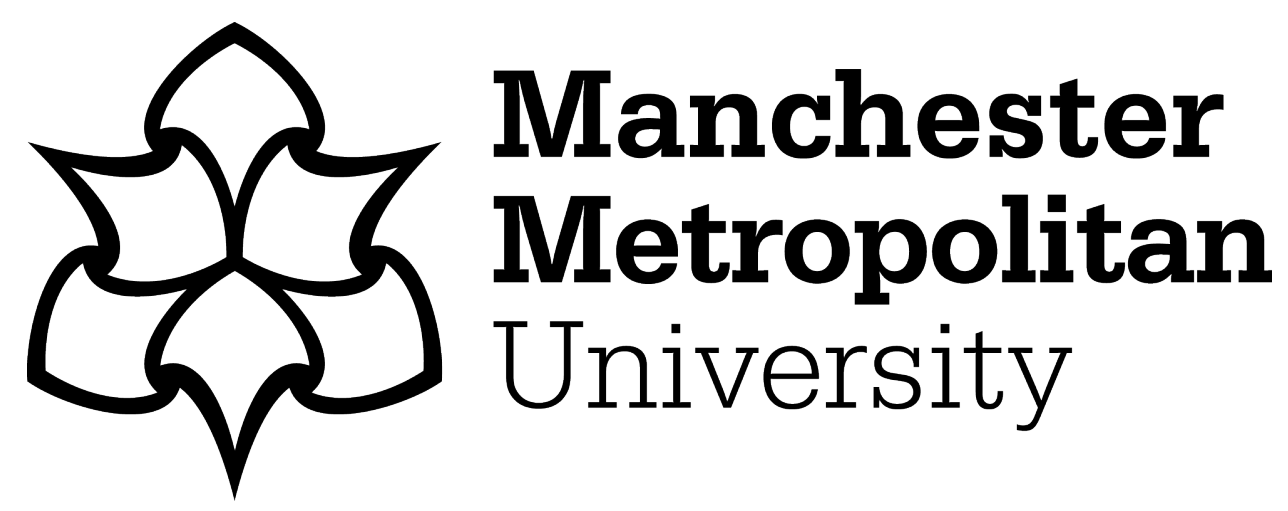

Adegbenjo, AO, Olubambi, PA, Potgieter, JH, Nsiah-Baafi, E and Shongwe, MB (2017) Interfacial Reaction During High Energy Ball Milling Dispersion of Carbon Nanotubes into Ti6Al4V. Journal of Materials Engineering and Performance, 26 (12). pp. 6047-6056. ISSN 1059-9495

Downloaded from: https://e-space.mmu.ac.uk/628320/

Version: Accepted Version

Publisher: Springer

DOI: https://doi.org/10.1007/s11665-017-3041-8

Please cite the published version 


\title{
Interfacial Reaction During High Energy Ball Milling Dispersion of Carbon Nanotubes into Ti6Al4V
}

\author{
A.O. Adegbenjo, P.A. Olubambi, J.H. Potgieter, E. Nsiah-Baafi, and M.B. Shongwe
}

(Submitted April 1, 2017; in revised form July 31, 2017; published online October 30, 2017)

\begin{abstract}
The unique thermal and mechanical properties of carbon nanotubes (CNTs) have made them choice reinforcements for metal matrix composites (MMCs). However, there still remains a critical challenge in achieving homogeneous dispersion of CNTs in metallic matrices. Although high energy ball milling (HEBM) has been reported as an effective method of dispersing CNTs into metal matrices, a careful selection of the milling parameters is important not to compromise the structural integrity of CNTs which may cause interfacial reactions with the matrix. In this study, multi-walled carbon nanotubes (MWCNTs) were purified by annealing in argon and vacuum atmospheres at 1000 and $1800{ }^{\circ} \mathrm{C}$, respectively, for $5 \mathrm{~h}$ to remove possible metallic catalyst impurities. Subsequently, 1, 2 and 3 wt.\% MWCNTs were dispersed by adapted HEBM into Ti6Al4V alloy metal matrix. Raman spectroscopy (RS), x-ray diffraction, scanning electron microscopy, energy-dispersive $x$-ray spectrometry and transmission electron microscopy techniques were used to characterize the as-received and annealed MWCNTs, as well as the admixed MWCNT/ Ti6Al4V nanocomposite powders. The experimental results showed that vacuum annealing successfully eliminated retained nickel (Ni) catalysts from MWCNTs, while the adapted HEBM method achieved a relative homogeneous dispersion of MWCNTs into the Ti6Al4V matrix and helped to control interfacial reactions between defective MWCNTs and the metal matrix.
\end{abstract}

Keywords aerospace, composites, metallic matrix, nanomaterials, powder metallurgy, titanium

\section{Introduction}

Titanium and its alloys are choice materials employed in the aerospace, automobile and chemical industries due to their low specific gravity, high specific strength and excellent corrosion resistance (Ref 1-3). They are very popular as competitive metal matrix materials in the aerospace and automotive industries because they can fulfill the increasing demand for high-performance engineering materials with light weight and high strength needed in these industries (Ref 4, 5). Among titanium alloys, Ti6Al4V (an $\alpha+\beta$ titanium alloy) is commonly referred to as the "workhorse" of both the titanium and the aerospace industries, as it is the most commonly used alloy in these industries mainly because of its comprehensive mechanical properties (Ref 6-9). In spite of the crucial importance of Ti6Al4V in these industries, its low hardness and poor tribological properties often limit its use as structural components (Ref 10).

A.O. Adegbenjo and P.A. Olubambi, Centre for Nanoengineering and Tribocorrosion, Faculty of Engineering and the Built Environment, University of Johannesburg, Johannesburg, South Africa; J. H. Potgieter, School of Chemical and Metallurgical Engineering, University of the Witwatersrand, Johannesburg, South Africa; and E. Nsiah-Baafi and M. B. Shongwe, Institute for NanoEngineering Research, Department of Chemical, Metallurgical and Materials Engineering, Tshwane University of Technology, Pretoria, South Africa. Contact e-mail: waleeleect@gmail.com.
The need for fuel economy, weight and cost savings has driven researchers into developing high-performance titanium (Ti)-based lightweight composite materials. These will serve as replacements for the unreinforced conventional $\mathrm{Ti}$ alloys often used for highly demanding structural applications and thus guarantee enhanced properties of interest (Ref 11, 12). The superior mechanical and thermal properties of carbon nanotubes (CNTs) have made them choice reinforcement materials for Ti metal matrix composites (TMCs) (Ref 13). These unique attributes of CNTs are associated with their seamless cylindrical morphology which entirely consists of $\mathrm{sp}^{2} \mathrm{C}-\mathrm{C}$ network (Ref $14,15)$. The presence of these $\mathrm{sp}^{2} \mathrm{C}-\mathrm{C}$ bonds in the outer shells of CNTs give them enormous chemical stability as these bonds are known to be among the strongest chemical bonds in nature (Ref 16, 17). These $\mathrm{sp}^{2} \mathrm{C}-\mathrm{C}$ bonds are formed into an hexagonal honeycomb lattice in the CNTs with closed edges having an interlayer spacing of $0.34 \mathrm{~nm}$ (Ref 14, 17). Three electrons out of the four valence electrons in $\mathrm{sp}^{2}$-hybridized carbon structures, such as CNTs, form covalent bonds with the in-plane neighboring electrons. The fourth electron is therefore allowed to be delocalized within the carbon atoms (Ref 18). Thus, $\mathrm{sp}^{2}$ hybridization in carbon structures forms strong inplane C-C bonds, but weaker out-of-plane bonds (Ref 17, 19).

Carbon nanotube-reinforced $\mathrm{Ti}$ metal matrix composites (CNT-TMCs) have been reported as promising replacements for the unreinforced Ti alloys (Ref 11, 12, 20, 21). However, a critical limitation with the fabrication of CNT-TMCs is the difficulty in achieving the homogeneous dispersion of CNTs in the Ti metal matrix (Ref 20-22). This challenge arises as a result of the nanosize dimensions of CNTs, their high aspect ratio, large surface area, characteristic tubular morphology and the strong van der Waals forces in the individual tubes which cause CNT agglomeration (Ref 11, 12, 23). In order to overcome these challenges, different studies have reported various dispersion methods for CNTs in Ti matrices. These include 
high energy ball milling (HEBM) (Ref 12, 22), surfactant coatings (Ref 4, 24), mechanical mixing (Ref 24, 25) and sonication-assisted ball milling (Ref 12). Among these methods, HEBM has been reported to be effective and economical for dispersing MWCNTs in metal matrices (Ref 26, 27). The technique has also been successfully used to disperse MWCNTs in Ti matrices specifically (Ref 3, 12, 22, 28). However, a carefully optimized choice of the milling parameters is crucial as harsh ball milling conditions promote severe damaging effects in the $\mathrm{sp}^{2} \mathrm{C}-\mathrm{C}$ network in CNTs (Ref 29, 30). Using a high milling energy (dictated by high milling speeds, high ball-to-powder ratios (BPRs), among other parameters) may increase the diffusivity of charged powders and hasten the mechanical alloying process by introducing structural defects and fractured surfaces (Ref 31). Extended milling times, high milling speeds and high BPRs can affect the quality and integrity of CNTs negatively, damaging their outer shells by introducing open ends. These open ends destroy the basal planes of the CNTs and expose the prismatic planes, where the favored growth of interfacial products (such as carbides) can occur (Ref 32). Vacancies and defects are also created in the CNTs side walls (Ref 33,34$)$, constituting non-sp ${ }^{2}$ disorders in them. The conversion of $\mathrm{sp}^{2}-$ to $\mathrm{sp}^{3}$-hybridized carbons not only changes the morphology of the CNTs, but also causes the loss of their unique properties of interest (Ref 12, 35). These highly reactive $\mathrm{sp}^{3} \mathrm{C}-\mathrm{C}$ amorphous phases are potential initiation sites for interfacial reactions with the Ti metal matrix during ball milling, resulting in the formation of titanium carbide (TiC) as the interfacial product (Ref 32,36$)$.

During ball milling, plastic deformation, cold welding and fracture are the three predominant activities taking place, as proposed by Yadav et al. (37). Plastic deformation brings about a change in the particle shape, while fracture leads to a decrease in powder particle size. The latter effect subsequently results in the formation of finely dispersed alloying particles in the grain-refined soft metal matrix powder. Cold welding, on the other hand, leads to an increase in particle size of the powder species (Ref 37). Cold welding is an unwanted event occurring during milling, especially with HEBM. Hence, process control agents (PCA) such as stearic acid (Ref 38, 39), ethanol (Ref 40, 41) and ethylene bis-stearamide (Ref 4244) are usually added to the powders being milled to reduce the possible occurrence of cold welding and instead promote fracturing (Ref 28, 45). These surfactants are commonly added in small quantities (usually $0.5-3 \mathrm{wt} . \%$ ) in order to reduce contamination within the charged powders (Ref 28). Stearic acid is the most commonly employed PCA during dry HEBM dispersion of CNTs in Ti matrices (Ref 3, 11, 12, 28, 32). Nevertheless, it has been reported that the PCA may decompose into its constituent elements (carbon, hydrogen and oxygen) under harsh and prolonged milling conditions. This in turn can result in cold welding, rather than promoting the fracturing of powder particles (Ref 38, 46). These elements become contaminations in the powders being milled (e.g., TMC powders) and thus lower the surface energy and initiate the formation of $\mathrm{TiC}$.

Although there have been some reported studies on CNTTMCs which focused on understanding the effect of milling energy and parameters on the synthesis of these materials (Ref 11, 32), the effect of a CNT dispersion method on the deterioration, interfacial reactions and re-agglomeration of CNTs (Ref 12), and the role of PCA on synthesis of these material powders (Ref 28 ), the incorporation of CNTs into a
Ti6A14V metal matrix have received little attention and were not reported to date. Therefore, the focus of this present study was aimed at overcoming the challenges of interfacial reactions and achieving homogeneous dispersion of CNTs in TMCs. This was accomplished by exploring the adaptation of the HEBM process as a mixing medium for the dispersion of multi-walled carbon nanotubes (MWCNTs) in the Ti6Al4V matrix, rather than it being used as a milling medium. It was endeavored to achieve neither size reduction, nor fracturing of the matrix powder. Mixing was performed without a PCA addition to eliminate the possibility of contamination often encountered with PCA decomposition into its constituent elements. The parameters were also chosen to avoid cold welding of the matrix alloy which usually occurs due to high frictional heat being generated during milling processes. The MWCNTs evolution during processing, their dispersion in the Ti6Al4V matrix and the formation of in situ crystalline TiC phase were quantitatively investigated. The results presented will form a useful basis and assist in the novel fabrication of lightweight, high performance, MWCNT-reinforced Ti6Al4Vbased MMCs for aerospace and automobile structural applications targeted for fuel economy and cost savings in these industries.

\section{Experimental}

\subsection{Starting Materials}

The MWCNTs used as reinforcement in this study were supplied by Cheap Tubes Inc., USA with specifications (outside diameter $(\mathrm{OD})=20-30 \mathrm{~nm}$, length $(L)=10-30 \mu \mathrm{m}$, ash $<1.5$ wt.\%, purity $>95$ wt.\%). The alloy (Ti6Al4V) metal matrix powder (argon atomized, with average particle size $-25 \mu \mathrm{m}$ ) was supplied in prealloyed condition by TLS Technik GmbH \& Co., Germany. The as-received MWCNTs were subjected to graphitization (high-temperature annealing) treatments for $5 \mathrm{~h}$ at high temperatures in argon $\left(1000^{\circ} \mathrm{C}\right)$ and vacuum $\left(1800^{\circ} \mathrm{C}\right)$ atmospheres in tube furnaces (Brother XD-1200NT, China and Webb 77 Natick MA, USA). The heating rate used in both cases was $10^{\circ} \mathrm{C} / \mathrm{min}$.

\subsection{Mixing and Dispersion of MWCNTs in the Ti6AI4V Matrix}

A planetary ball mill (Retsch PM 400 MA, Germany) that is usually employed for HEBM was adapted for the mixing and dispersion of purified MWCNTs in Ti6Al4V metal matrix. Amounts of 1,2 and 3 wt.\% MWCNTs were dispersed into the metal matrix by mixing in the ball mill for $6 \mathrm{~h}$ at a rotational speed of $50 \mathrm{rpm}$. Both the matrix alloy and the predetermined wt.\% MWCNT reinforcement were charged into stainless steel vials $(250 \mathrm{ml}$ capacity and $80 \mathrm{~mm}$ inner diameter $\left(\mathrm{I}_{\mathrm{D}}\right)$ ) along with stainless steel balls of three different sizes (diameter $(d)=3,5$ and $7 \mathrm{~mm}$ ) in a weight ratio of $1: 3: 1$. The essence of using three ball sizes was to prevent the cold welding of charged powders and to increase the collision energy available to the powder particles (Ref 45, 47). Dry mixing of the charged powders was carried out without the addition of a PCA to avoid powder contamination in the event of breakdown of the PCA into its constituent elements during the mixing process. The ball-to-powder ratio was set at 10:1 while the ball mill was programmed to rest for $10 \mathrm{~min}$ after every $10 \mathrm{~min}$ of mixing, so that the powders will 
not be heated up due to excessive frictional and impact forces from ball-powder-ball interactions.

\subsection{Characterization of Powders}

All the starting powders (as-received and purified MWCNTs, the Ti6Al4V metal matrix powders) and the admixed MWCNTs/Ti6Al4V nanocomposite powders were characterized by scanning electron microscope equipped with energy-dispersive $\mathrm{x}$-ray spectrometry (EDX) facilities (JEOL JSM-7600F, Japan and Zeiss Ultra Plus, Germany) and transmission electron microscopy (JEOL JEM-2100F) to study the microstructure and surface morphologies of the powders. The structural changes and crystalline phases in the admixed composite powders were also studied using x-ray diffractometer (PANalytical Empyrean model) with $\mathrm{Cu} \mathrm{K \alpha}$ radiation $(\lambda=0.154 \mathrm{~nm})$ at a scan rate of $1^{\circ} / \mathrm{min}$ over $10^{\circ}-90^{\circ}$ angular range and was analyzed with Highscore plus software. Raman spectroscopy (T6400 Jobin-Yvon, HORIBA, Japan) was carried out on the as-received matrix and reinforcement starting powders, as-purified MWCNTs and the admixed composite powders with a $514.5 \mathrm{~nm}$ laser employing a $20 \times$ objective lens. Raman scattering was obtained on the powder samples with an acquisition time of $30 \mathrm{~min}$. The spectra range of 1200$2000 \mathrm{~cm}^{-1}$ and $200-1800 \mathrm{~cm}^{-1}$ were used for purified MWCNTs and the admixed nanocomposite powders, respectively.

\section{Results and Discussion}

\subsection{Morphology of Starting Powders}

The morphology of the as-received Ti6Al4V and MWCNTs starting powders is presented in Fig. 1. The Ti6Al4V matrix powder has spherical and near spherical-shaped particles (Fig. 1a), typical of a gas-atomized process. No visible evidence of agglomeration in seen in the powders. The asreceived MWCNTs are strongly agglomerated into large clusters (Fig. 1b) and highly entangled into one another (Fig. 1c). These features are due to the strong van der Waal forces of the individual tubes in the CNT powders, their large surface areas and high aspect ratios (Ref 12,48). The inset in Fig. 1(c) is the TEM image of the agglomerated MWCNTs having black spots within and outside the nanotubes. These dark spots have been identified previously by Feng et al. (49) to be retained transition metal catalysts, amorphous carbon and carbonaceous nanoparticles. This is as a result of the catalytic chemical vapor deposition (CCVD) process by which the MWCNTs were produced. Figure 1(d) is the SEM image of an individual MWCNT showing its tubular structure.

\subsection{Preparation of As-Received MWCNTs}

Iron $(\mathrm{Fe})$ and nickel $(\mathrm{Ni})$ are the most frequently encountered metallic catalyst elements that are found retained in synthesized CNTs owing to the use of ferrocene and nickelocene catalysts, respectively, during CNT production (Ref 50). Figure 2(b) is the EDX analysis result of the as-received MWCNT (Fig. 2a) used in this study. Only Ni was found retained in the MWCNTs, but not Fe. Other metallic elements such as aluminum (Al) and copper $(\mathrm{Cu})$ maybe present in synthesized CNTs, even after purification (Ref 50), as seen in
Fig. 2(b). However, their presence is not much of a concern compared to that of $\mathrm{Fe}$ or $\mathrm{Ni}$ because they do not form intermetallic compounds that are stable at high temperatures. Intermetallic compound phases are seldom desired in composites as their brittleness could compromise the ductility and toughness characteristics of the bulk composite material (Ref 51).

Many of the methods used for the synthesis of CNTs make use of metal catalysts which remain trapped in the final CNTs produced (Ref 50). Aside from these retained metal catalysts, the as-synthesized CNTs also do contain carbon nanoparticles, amorphous carbon and some structural defects (Ref 52, 53). The purification of the as-synthesized CNTs is therefore necessary as these defects and impurities affect the properties of CNTs and accelerate their decomposition. This may lead to the degrading or total loss of the choice properties of interest needed in various applications (Ref 50, 52, 53). Acid washing and graphitization treatments are the commonly used methods for the purification of CNTs (Ref 53). However, the latter is the most effective for eliminating both retained catalyst particles and the structural defects in the CNTs (Ref 52). On the other hand, further impurities are introduced to CNTs during severe acid washing, and this may damage the side walls of the CNTs and compromise the integrity of the length and perfection of the nanotubes, although some structure may be reclaimed by annealing (Ref 49, 50).

The as-received MWCNTs in this study were subjected to high-temperature annealing in tube furnaces in argon and vacuum atmospheres at 1000 and $1800{ }^{\circ} \mathrm{C}$, respectively. Figure 3 shows the SEM images of MWCNTs after annealing them for $5 \mathrm{~h}$ in the respective atmospheres and temperatures. The corresponding EDX analysis results are presented in Fig. $3\left(a^{1}\right)$ and $\left(b^{1}\right)$, respectively. The retained Ni catalyst was successfully removed after vacuum annealing at $1800{ }^{\circ} \mathrm{C}$. The thermodynamic stability of CNTs is reported to be improved by high-temperature thermal annealing due to the elimination of dangling bonds plus other structural defects, thereby forming more refined graphitic structures (Ref 52). To evaluate this phenomenon, the annealed MWCNTs were tested by Raman spectroscopy and the $\mathrm{I}_{\mathrm{D}} / \mathrm{I}_{\mathrm{G}}$ ratio was used as the comparative characterization basis.

This has been reported to correspond to the graphitization or degree of crystallinity of MWCNTs and has been a useful tool for the qualitative and quantitative evaluation of MWCNTs evolution during processing (Ref 11, 28). The Raman spectra of the as-received and annealed MWCNTs are displayed in Fig. 4. The spectrum for the as-received MWCNTs (Fig. 4a) showed two characteristic peaks at 1344 and $1565 \mathrm{~cm}^{-1}$, respectively. These correspond to the disordered D and graphitic G bands, respectively (Ref 54, 55). The D band in MWCNTs represents the concentration of defects and is related to the $\mathrm{C}-\mathrm{C}$ bond disorders referred to as the non- $\mathrm{sp}^{2}$ structural defects in graphitic materials (Ref 54), while the $\mathrm{G}$ band describes the in-plane C-C bond stretching mode in MWCNTs known as the degree of metallicity or crystallinity in graphitic materials (Ref 28, 56).

The Raman peak $\left(\mathrm{I}_{\mathrm{D}} / \mathrm{I}_{\mathrm{G}}\right)$ ratio for the as-received MWCNTs was 1.30. However, this ratio was reduced to 0.90 after the MWCNTs were annealed in argon at $1000{ }^{\circ} \mathrm{C}$ indicating a reduction of about $31 \%$ in the measured Raman peak ratio. This suggests that the structural integrity of the MWCNTs was improved after annealing them in argon. The D and G shifts also changed to 1341 and $1568 \mathrm{~cm}^{-1}$, respectively. The 

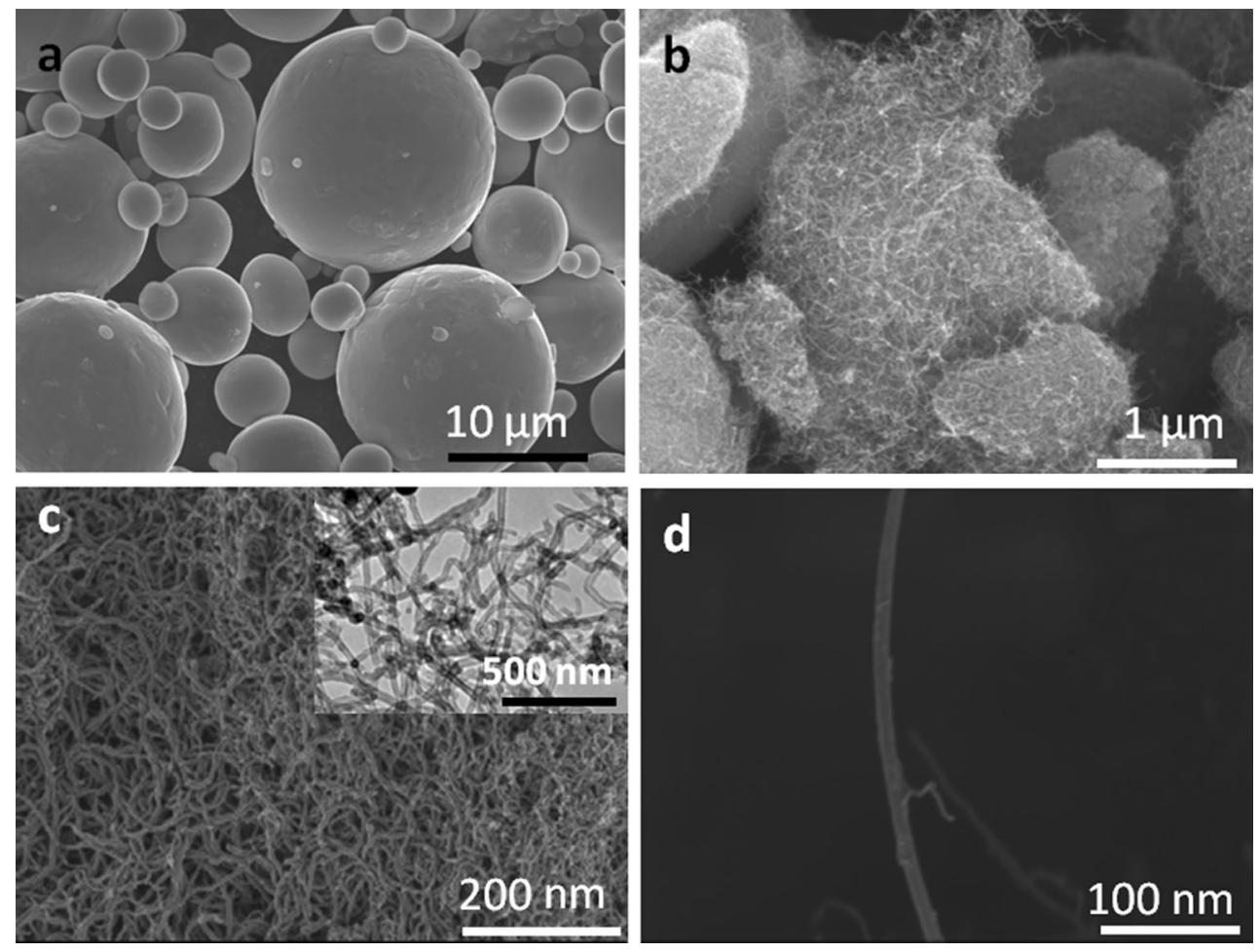

Fig. 1 SEM and TEM images of starting powders: (a) Ti6Al4V, (b) low-magnification SEM image of clustered and agglomerated MWCNTs, (c) high-magnification SEM image of entangled MWCNTs with the TEM image as inset, (d) SEM image of individual MWCNT
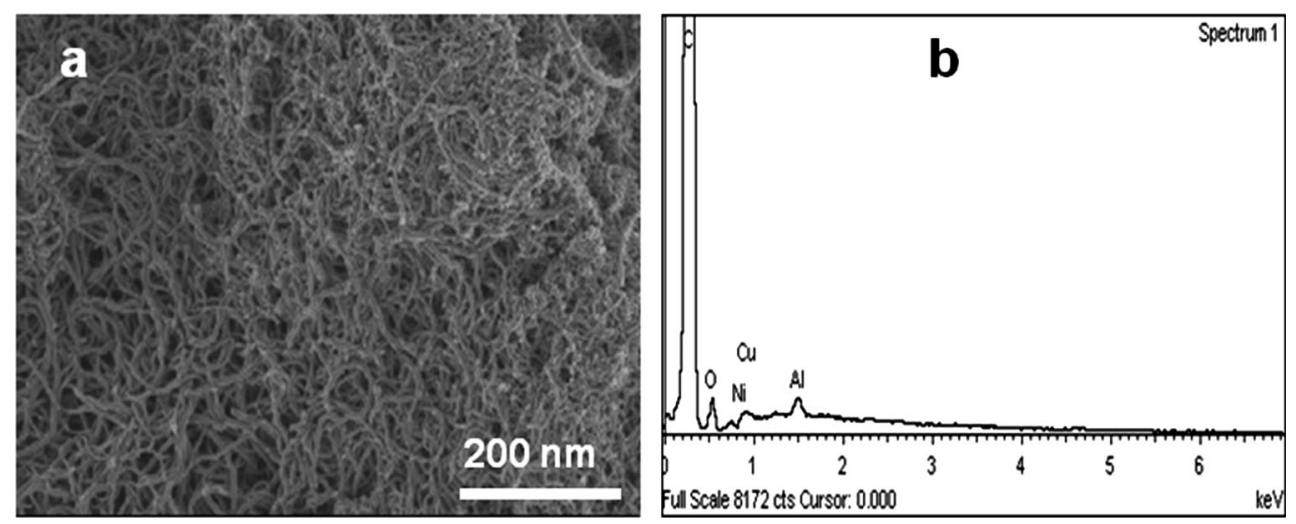

Fig. 2 SEM image of (a) as-received MWCNTs and (b) corresponding EDX analysis of the as-received MWCNTs

MWCNTs annealed in vacuum at $1800{ }^{\circ} \mathrm{C}$ (hereafter referred to as MWCNTsv) had a $I_{\mathrm{D}} / I_{\mathrm{G}}=0.8$, which suggests the dominance of $\mathrm{sp}^{2} \mathrm{C}-\mathrm{C}$ bonds over the non-sp ${ }^{2}$ disorders (Ref 28). This corresponds to reductions of 39 and $11 \%$ in the $I_{\mathrm{D}} / I_{\mathrm{G}}$ ratio of the as-received and argon annealed MWCNTs, respectively. The $D$ and $G$ bands nevertheless shifted to 1340 and $1570 \mathrm{~cm}^{-1}$, respectively. The observed shifting of the $\mathrm{G}$ bands in the annealed MWCNTs to higher wave numbers is evidence of induced stresses to the MWCNTs during processing.

These results are in agreement with the EDX reports presented earlier and thus confirm that the as-received MWCNTs were effectively purified by annealing it in vacuum at $1800{ }^{\circ} \mathrm{C}$. It was also observed that the $\mathrm{G}$ band peaks were narrower with graphitization treatments. The MWCNTsv showed a narrower peak suggesting a better refinement of the structure and increased crystallinity of the nanotubes. The structural refinement to ordered MWCNTs from the disordered form, accompanied by crystallite growth, has been proposed to follow three processes: (a) straightening and rearrangement of distorted small graphene layers, (b) fusion between the graphene layers and (c) development to larger graphene layers along the nanotube axis and removal of stacking faults between graphene layers in a restricted space (Ref 52, 57).

\subsection{XRD Analysis of As-Received Ti6Al4V, MWCNTsv and Admixed MWCNTsv/Ti6AI4V Nanocomposite Powders}

Figure 5 shows the XRD patterns of the MWCNTsv, asreceived Ti6Al4V and the admixed composite powders containing 1, 2 and 3 wt.\% of the MWCNTsv used as the reinforcement powder in the Ti6Al4V alloy matrix. The powders were mixed for $6 \mathrm{~h}$ in the planetary ball mill. The 

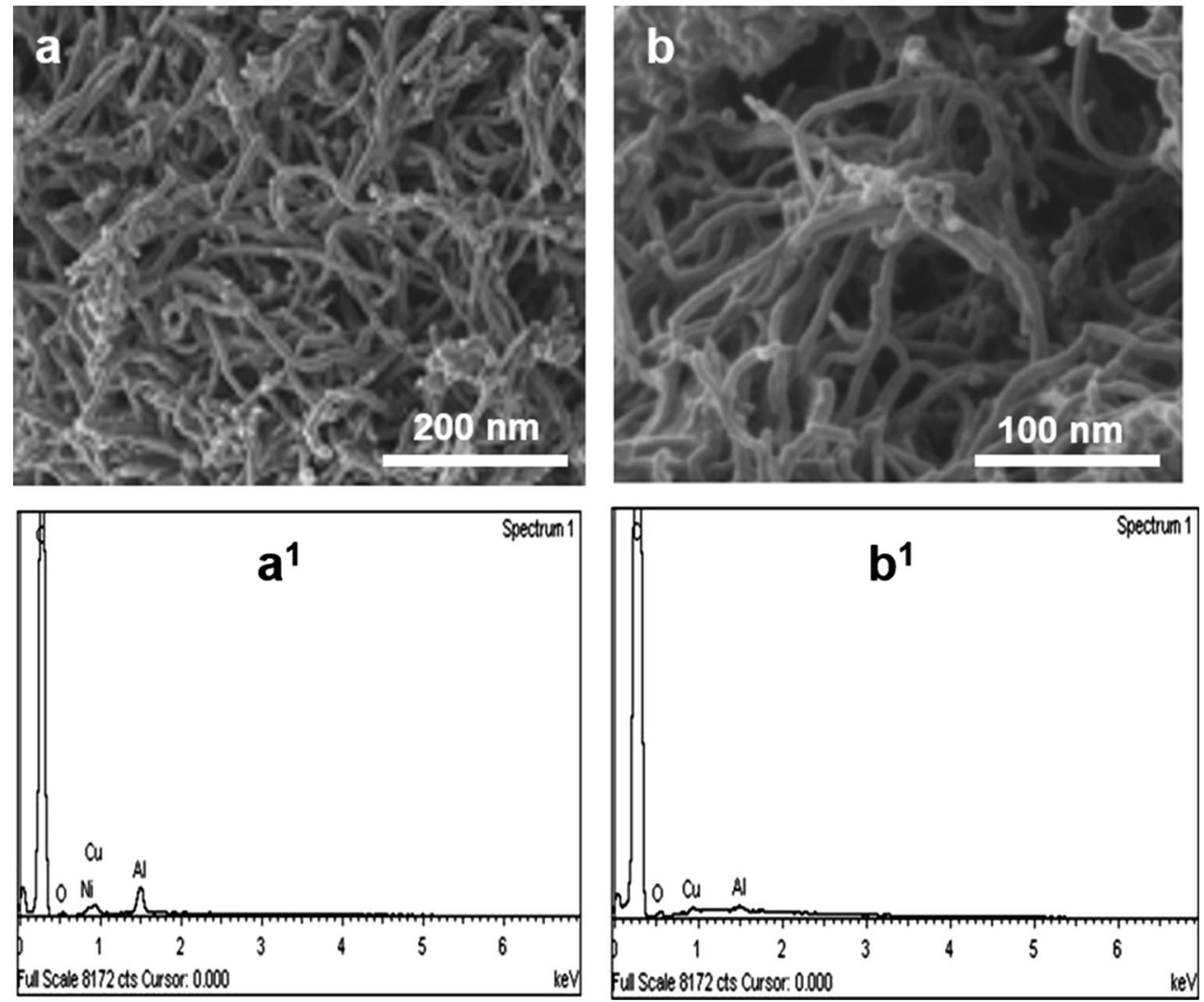

Fig. 3 SEM images of annealed MWCNTs (a) annealed in argon at $1000{ }^{\circ} \mathrm{C}$ (b) annealed in vacuum at $1800{ }^{\circ} \mathrm{C}$ and the corresponding EDX results in $\left(a^{1}\right)$ and $\left(b^{1}\right)$, respectively

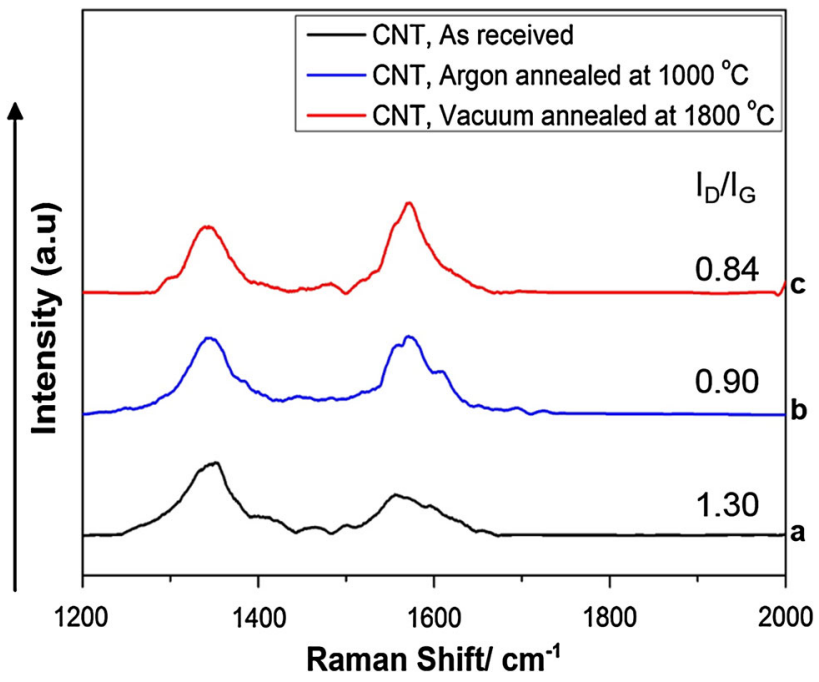

Fig. 4 Raman spectra of MWCNTs (a) as-received, (b) annealed in argon at $1000{ }^{\circ} \mathrm{C}$, (c) annealed in vacuum at $1800{ }^{\circ} \mathrm{C}$

XRD patterns of the admixed nanocomposite powders showed only $\alpha$-Ti peaks with no evidence of peak broadening, suggesting that there was no reduction in the sizes of the matrix Ti6A14V powder particles. This further confirms that the objective of adapting the high energy planetary ball mill for mixing was achieved. No MWCNTs peaks were detected in the composite powders. This could be due to the limitation of the

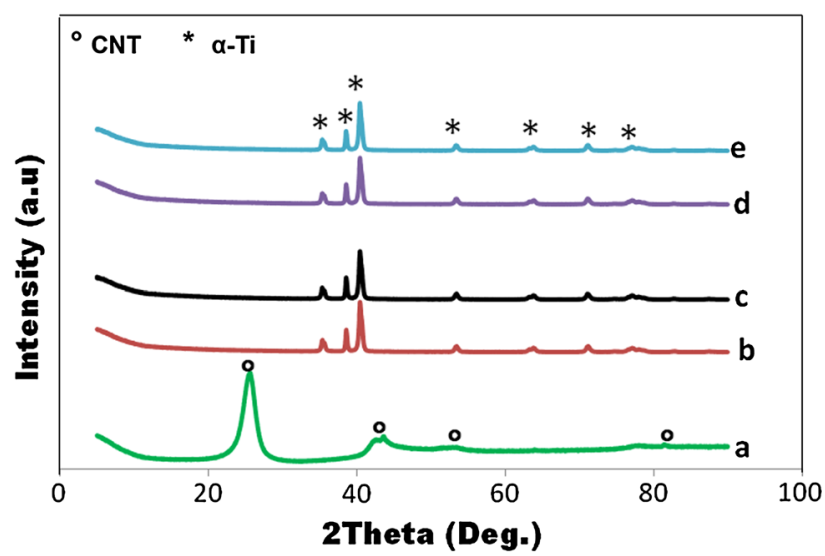

Fig. 5 XRD patterns of MWCNTsv/Ti6Al4V admixed composite powders (a) MWCNTsv, (b) as-received Ti6Al4V, (c) Ti6Al4V1\%MWCNTsv (d) Ti6A14V-2\%MWCNTsv, (e) Ti6Al4V-3\%MWCN Tsv

XRD technique in not being able to detect the small fractions of MWCNT in the Ti6Al4V matrix powders. It has also been reported that owing to the wide variation in the mass absorption coefficients (for $\mathrm{Cu} \mathrm{K} \alpha$ radiations) of carbon (C) and Ti which are, respectively, 4.6 and $208 \mathrm{~m}^{2} \mathrm{~g}^{-1}$, it is often difficult for $\mathrm{XRD}$ to detect $\mathrm{C}$ in the presence of Ti (Ref 58, 59). However, as previously explained by Cai et al. (Ref 60), the absence of MWCNT peaks in the XRD patterns may as well possibly be as 
a result of the homogeneous dispersion of the MWCNTsv in the Ti6Al4V matrix and the amorphization of the MWCNTsv.

\subsection{Raman Spectroscopy of the Matrix, Reinforcement and MWCNT/Ti6AI4V Nanocomposite Powders}

The Raman spectra of the as-received Ti6Al4V matrix alloy, MWCNTsv and the admixed composite powders are presented in Fig. 6. It is beneficial to quantitatively characterize the evolution of MWCNTs during composite processing, so as to develop and monitor the relationship between the mechanical properties of the final bulk composite and the processing parameters (Ref 28).

As observed from Fig. 6(a), the Raman spectra for asreceived Ti6Al4V powder do not show any peaks. This is because $\mathrm{Ti}$, as noticed for most metals, does not exhibit active Raman vibrational modes (Ref 61). The MWCNTsv as previously stated had two main peaks at 1340 and $1570 \mathrm{~cm}^{-1}$, corresponding to the $\mathrm{D}$ band and $\mathrm{G}$ band, respectively, with a characteristic $I_{\mathrm{D}} / I_{\mathrm{G}}$ ratio $=0.8$. The Raman spectra for the composite powder containing $1 \mathrm{wt} . \%$ MWCNTsv (Fig. 6c) had its D and G bands, respectively, at 1342 and $1581 \mathrm{~cm}^{-1}$. The graphitic bands had shifted to upper wave numbers, indicating an induced stress on the MWCNTs during the composite mixing (Ref 28,29$)$. The $I_{\mathrm{D}} / I_{\mathrm{G}}$ ratio was increased to 1.01 , translating to a $26 \%$ increase as compared with the as annealed MWCNTsv without Ti6Al4V. Also, the composite powder with 2 wt. $\%$ MWCNTsv had a $I_{\mathrm{D}} / I_{\mathrm{G}}=0.97$, which is a $21 \%$ increase from 0.8 for the annealed MWCNTsv. However, for the composite powder with $3 \mathrm{wt} . \%$ MWCNTsv, the wave numbers for the $D$ and $G$ bands could not be determined as these peaks had disappeared due to the amorphization of the MWCNTsv.

A progressive broadening of the $\mathrm{D}$ and $\mathrm{G}$ bands was observed with increasing amounts of the wt.\% MWCNTsv in the Ti6Al4V matrix. However, as the $\mathrm{G}$ band corresponds to the degree of graphitic arrangement and crystallinity of the Catoms in the graphitic network of the MWCNTs, any broadening of this Raman peak is an indication of the presence of induced disorders or loss of crystallinity (Ref 61). Thus, as proposed by Munir et al. (Ref 11), it can be inferred that the occurrence of defect concentrations and extensive loss of crystallinity, in addition to amorphization, increased with

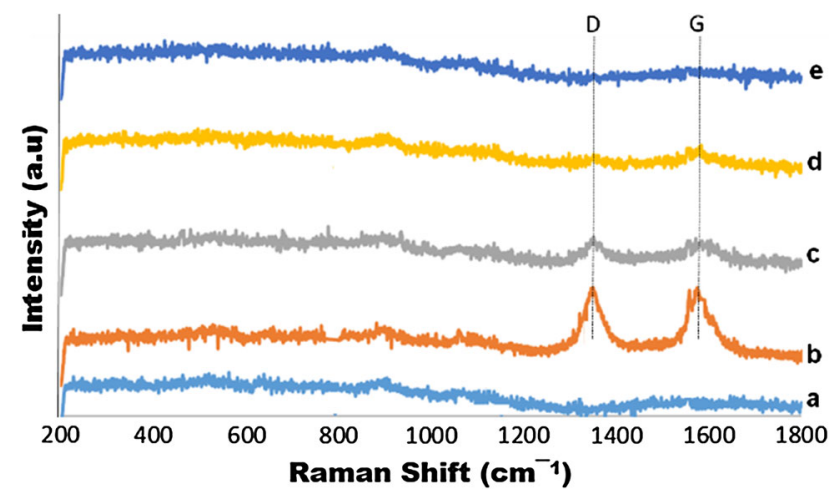

Fig. 6 Raman spectra of as-received Ti6Al4V, annealed MWCNT and MWCNT/Ti6Al4V admixed composite powders (a) as-received Ti6Al4V, (b) MWCNTsv, (c) Ti6Al4V-1\%MWCNTsv, (d) Ti6A14V2\%MWCNTsv, (e) Ti6A14V-3\%MWCNTsv. (Note: The D and G bands in MWCNTs represent the defects concentration and degree of metallicity or crystallinity, respectively) increased wt.\% MWCNTsv additions in the Ti6A14V matrix during the mixing of the composite powders. Also, the observed decrease in the $\mathrm{G}$ band peak intensities with increased wt.\% MWCNTsv is attributed to the presence of non-sp ${ }^{2}$ structural defects in $\mathrm{C}-\mathrm{C}$ bonds of the graphitic structure of MWCNTsv owing to mixing (Ref 12). During mixing, a deagglomeration of the clustered and agglomerated MWCNTsv occurs. This results in a dispersion of the individual MWCNTsv in the Ti6Al4V metal matrix in such a way that the collision energy from the balls is mostly absorbed by the matrix powders. Due to this, the structural damage in MWCNTsv, due to limited direct impact energy being received by the reinforcement CNT powders, is reduced. Nevertheless, as the wt.\% MWCNTsv increased in the metal matrix, the degree of deagglomeration and dispersion of individual MWCNTsv is reduced. Therefore, as the mixing process progressed, individual MWCNTsv in the agglomerated MWCNTsv interacts with its immediate hard neighbor through impact, friction and deformation; consequently the energy generated during mixing via ball-powder-ball interaction is now primarily absorbed by individual MWCNTsv, thus resulting in the formation of non$\mathrm{sp}^{2}$ structural defects. The consequential effect of this was the observed total loss of crystallinity in the composite powder with 3 wt.\% MWCNTsv as seen in Fig. 6(e).

\subsection{SEM Morphologies of Admixed MWCNTsv/Ti6Al4V Nanocomposite Powders}

The morphologies of the admixed MWCNTsv/Ti6Al4V nanocomposite powders after $6 \mathrm{~h}$ mixing in the planetary ball mill are presented in Fig. 7. The relatively homogeneously dispersed MWCNTsv powders can be seen coating the Ti6Al4V particles (dark spots indicated by arrows (Fig. 7a, b and c). No evidence of cold welding is seen in the metal matrix alloy, and their particles maintained their original sizes and structure. No fracturing of the particles occurred. This affirms that the dispersion process was actually by mixing, but not milling. The powder mixture with 1 wt.\% MWCNTsv (Fig. 7a) showed the best homogeneous dispersion of the MWCNTsv at the reinforcement CNT powders which lightly coated the Ti6Al4V matrix particles. However, as the wt.\% MWCNTsv increased in the matrix alloy, the degree of dispersion of the MWCNTsv decreased, leading to agglomerates of the reinforcement powder heavily coating the matrix powder particles (Fig. $7 \mathrm{~b}$ and c). This observation could be explained from the fact that at an increased wt.\% of the reinforcing MWCNTsv, the strong van der Waal forces between individual nanotubes are increased, thus increasing the tendency for the nanotubes to agglomerate into clusters as seen.

This observation is better appreciated in the corresponding SEM images taken at higher magnifications (Fig. $7 a^{1}, b^{1}$ and $\mathrm{c}^{1}$ ). The unbundled, individual MWCNTsv are finely dispersed in the Ti6Al4V alloy matrix in Fig. $7\left(\mathrm{a}^{1}\right)$ with no agglomerates or clusters, but with increased wt.\% MWCNTsv in the metal matrix, large agglomerates of MWCNTsv are seen. These are indicated as point " $X$ " in Fig. $7\left(b^{1}\right)$ and $\left(c^{1}\right)$, although a few unbundled MWCNTsv are still dispersed in the matrix alloy.

\subsection{TEM Observation of Admixed Nanocomposite Powder Mixtures}

The results of the TEM observations on admixed nanocomposite powders are presented in Fig. 8. For the powder with 1 wt.\% MWCNTsv in the Ti6A14V alloy matrix, the MWCNTsv 

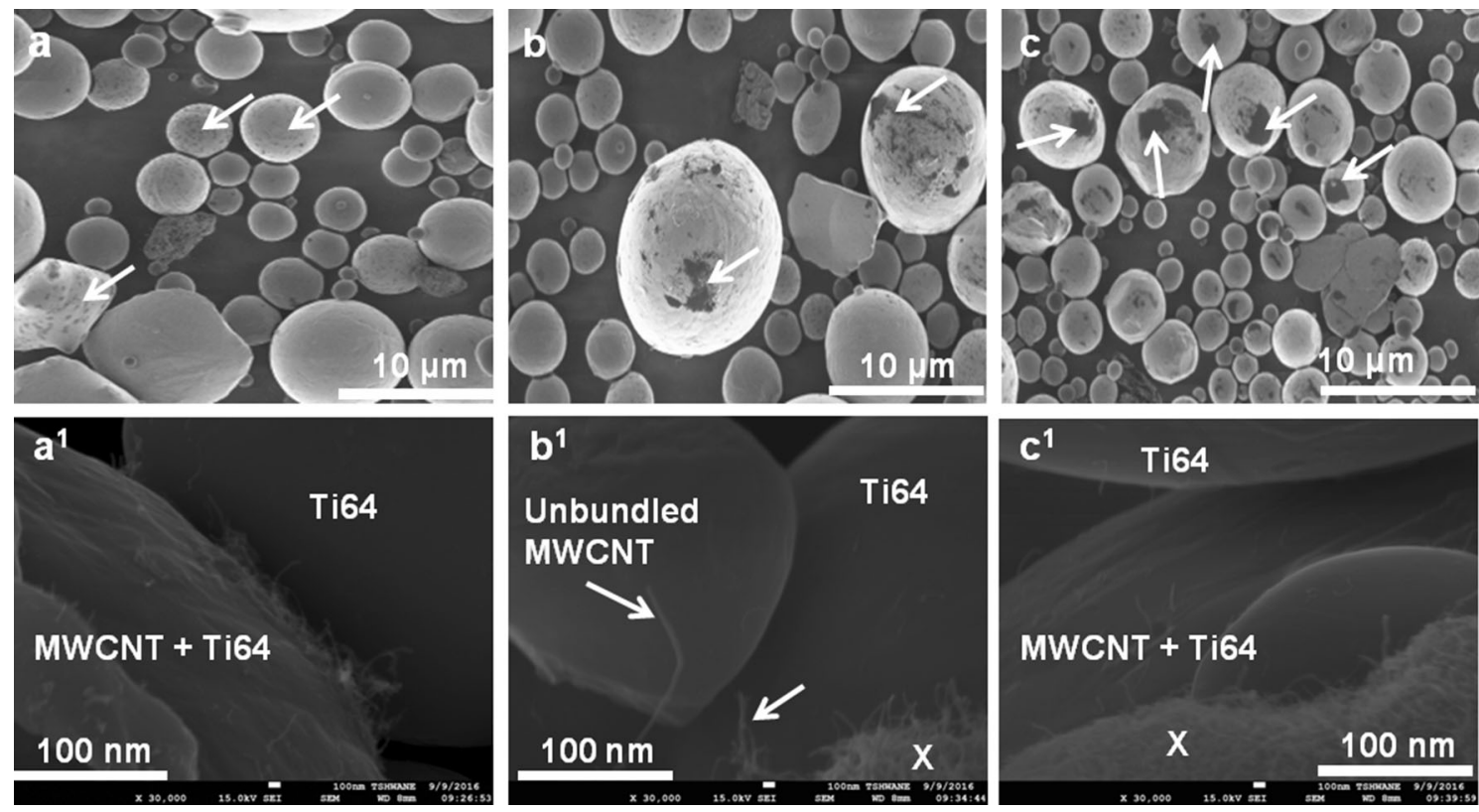

Fig. 7 SEM morphologies of MWCNTsv/Ti6Al4V admixed nanocomposite powders at low magnifications (a) Ti6Al4V-1\%MWCNTsv, (b) Ti6A14V-2\%MWCNTsv, (c) Ti6A14V-3\%MWCNTsv and the corresponding surface morphologies at high magnifications $\left(\mathrm{a}^{1}\right)-\left(\mathrm{c}^{1}\right)$

were well dispersed in the metal matrix as seen in Fig. $8\left(\mathrm{a}^{1}\right)$, $\left(\mathrm{a}^{2}\right)$ and $\left(\mathrm{a}^{3}\right)$, but some deformed MWCNTsv were observed (indicated with arrows) in Fig. $8\left(\mathrm{a}^{3}\right)$. These structural defects are either open edges or vacancies in the $\mathrm{C}-\mathrm{C}$ network which form due to absorbed energy from the balls and frictional forces among neighboring Ti6A14V particles and MWCNTsv (Ref 28, 32). The defect sites are potential areas for the formation of interfacial products, such as $\mathrm{TiC}$, owing to the interaction between the non-sp ${ }^{2}$ carbon defects and the Ti6Al4V matrix powders as defective MWCNTs are extremely reactive (Ref 32). However, no interfacial product was present, despite the observed defected MWCNTsv sites. It thus follows that the mixing parameters used in this work did not provoke the interaction between MWCNTsv and Ti6Al4V particles that could result in the formation of interfacial products at this wt.\% of the reinforcement powder in the matrix alloy. This is in agreement with the XRD and Raman analysis results discussed earlier.

However, as the wt. $\%$ of MWCNTsv increased to $2 \mathrm{wt.} \%$ in the matrix powder (Fig. $8 \mathrm{~b}^{1}$ and $\mathrm{b}^{2}$ ), a large agglomerate of MWCNTsv is seen in the matrix alloy (Fig. $8 b^{1}$ ). Also, Fig. $8\left(\mathrm{~b}^{2}\right)$ shows the MWCNTsv in a large Ti6Al4V particle. This suggests that the degree of dispersion of the CNT reinforcement powders in the metal matrix reduced with the increased wt.\% of the MWCNTsv in the matrix. Figure $8\left(b^{3}\right)$ shows part of the MWCNTsv was deformed; nevertheless, individual nanotubes still maintained their tubular structure after the nanocomposite powder mixing process. This is essential in order to ensure that the properties of interest in nanotubes are not lost. Otherwise, the properties and integrity of the final bulk composite will be compromised.

The TEM micrographs in Fig. $8\left(\mathrm{c}^{1}\right),\left(\mathrm{c}^{2}\right)$ and $\left(\mathrm{c}^{3}\right)$ were taken from the composite powders containing 3 wt. $\%$ MWCNTsv in the metal matrix alloy. Figure $8\left(\mathrm{c}^{1}\right)$ shows MWCNTsv agglomerate in the Ti6A14V matrix. Although the MWCNTsv seem well dispersed within the matrix in Fig. $8\left(\mathrm{c}^{2}\right)$ and $\left(\mathrm{c}^{3}\right)$, TiC formation was observed as indicated by the arrows in
Fig. $8\left(\mathrm{c}^{3}\right)$. This observation implies that the non-sp ${ }^{2}$ defect sites created in the MWCNTsv reinforcement during mixing at this wt.\% MWCNTsv in Ti6A14V had favorable conditions to promote their reaction with the matrix alloy, thus enhancing the formation of in situ TiC interfacial products. This is contrary to what was observed for the composite powder with 1 wt.\% MWCNTsv, where the in situ TiC formation was absent, despite the defective sites in the MWCNTsv. It can thus be inferred that the higher the amount of the MWCNTsv powder in the Ti6Al4V metal matrix, the higher the possibility of having more clustered and agglomerated MWCNTsv within the matrix powder as the mixing process progressed due to the difficulty of dispersing the MWCNTsv homogeneously in the composite. This has the tendency to increase the frictional heat generated during mixing, due to the interaction between the metal matrix and MWCNTsv on one hand and between individual hard MWCNTsv among themselves. Consequently, these prevailing conditions were able to initiate the reaction between the defective MWCNTsv and the metal particles which led to the formation of the observed in situ TiC. Another favorable condition for the formation of the interfacial products is the increased tendency for amorphization of the MWCNTsv as its wt.\% in the matrix powder is increased. This has been discussed earlier with the Raman spectra of the admixed composite powders in Fig. 6. However, the Raman spectra did not show any peak corresponding to TiC. The reason for this could not be fully established. However, it may possibly be as a result of having fine $\mathrm{TiC}$ particles sparsely distributed inhomogeneously in the composite powders.

This paper concentrated on investigating the occurrence of interfacial reactions during HEBM dispersion of carbon nanotubes into Ti6Al4V and reported the characterization of the phases and structure in the ball-milled MMC powder mix. Subsequent work investigated the effect of MWCNTsv addition on the tribological properties of the spark plasma sintering (SPS)-consolidated MWCNTsv/Ti6Al4V MMCs. The influence of MWCNTsv and sintering temperature on the densifi- 

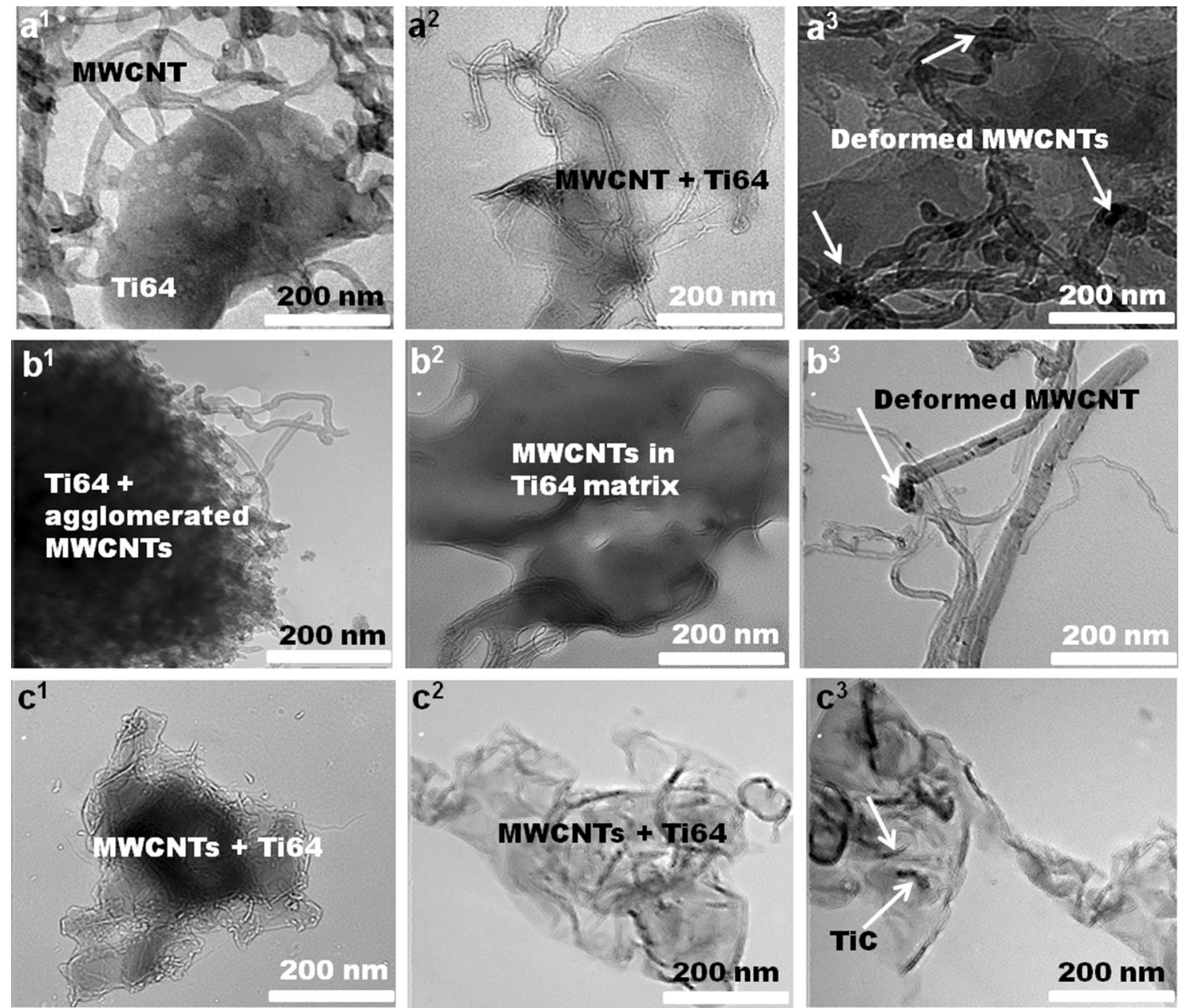

Fig. 8 TEM micrographs of MWCNTsv/Ti6Al4V admixed nanocomposite powders with varied wt.\% MWCNTsv in Ti6Al4V matrix powders: $\left(a^{1}\right)-\left(a^{3}\right)$ Ti6Al4V-1\%MWCNTsv, $\left(b^{1}\right)-\left(b^{3}\right)$ Ti6Al4V-2\%MWCNTsv, $\left(c^{1}\right)-\left(c^{3}\right)$ Ti6A14V-3\%MWCNTsv

cation and hardness behavior of the bulk composites was also studied. The findings are described in a follow-up paper under review elsewhere. Very briefly, it was found that the relative density of the synthesized MWCNTsv/Ti6Al4V composites was improved with increased sintering temperature, but deteriorated with an increased MWCNTsv weight fraction in Ti6Al4V. Nevertheless, the Vickers microhardness of the composites was enhanced with increased sintering temperature and weight fractions of MWCNTsv over that of the unreinforced Ti6Al4V. The MMCs also possessed superior wear resistances as well as outstanding improvement in the measured friction coefficients.

\section{Conclusion}

Interfacial reactions during the dispersion of carbon nanotubes into Ti6Al4V alloy matrix were investigated in this study. HEBM was adapted for the mixing and dispersion of 1, 2 and $3 \mathrm{wt} \%$ MWCNTsv in Ti6Al4V metal matrices, respectively. Although the adapted HEBM route was effective in homogeneously dispersing MWCNTsv into the alloy metal matrix, the level of dispersion reduced with the increase in the weight fraction of the MWCNTsv. Non-sp ${ }^{2}$ defects were created in MWCNTsv during mixing, and Raman analysis showed amorphization of MWCNTsv increased with increasing wt.\% MWCNTsv in the nanocomposite powders. A shift to the higher wave numbers and peak broadening were also observed in the characteristic $G$ bands of the MWCNTsv, which are indications of induced stresses and loss of crystallinity cum formation of highly reactive $\mathrm{sp} 3$ amorphous phases in the MWCNTsv, respectively. The formation of $\mathrm{TiC}$ interfacial product was dependent on the wt.\% of MWCNTsv as interfacial reaction between the defective MWCNTsv and Ti6Al4V was only triggered at 3 wt.\% MWCNTsv.

\section{Acknowledgments}

The authors are grateful to Tshwane University of Technology for offering Mr. Adegbenjo a postgraduate bursary award toward his doctoral study and Institute for NanoEngineering Research (INER) for the conducive laboratory environment in support of this research. The financial assistance from the National Research Foundation is also acknowledged.

\section{Conflict of interest}

The authors declare that they have no conflict of interest. 


\section{References}

1. C. Leyens and M. Peters, Titanium and Titanium Alloys. Fundamentals and Applications, Willey, ed., VCH Verlag $\mathrm{GmbH} \&$ Co. KGaA, Weinheim, 2003

2. J.C. Williams and E.A. Starke, Progress in Structural Materials for Aerospace Systems, Acta Mater., 2003, 51(19), p 5775-5799

3. K.S. Munir, Y. Zheng, D. Zhang, J. Lin, Y. Li, and C. Wen, Microstructure and Mechanical Properties of Carbon Nanotubes Reinforced Titanium Matrix Composites Fabricated via Spark Plasma Sintering, Mater. Sci. Eng. A, 2017, 688, p 505-523

4. F. Xue, S. Jiehe, F. Yan, and C. Wei, Preparation and Elevated Temperature Compressive Properties of Multi-walled Carbon Nanotube Reinforced Ti Composites, Mater. Sci. Eng. A, 2010, 527(6), p 15861589

5. K. Kondoh, T. Threrujirapapong, J. Umeda, and B. Fugetsu, HighTemperature Properties of Extruded Titanium Composites Fabricated from Carbon Nanotubes Coated Titanium Powder by Spark Plasma Sintering and Hot Extrusion, Compos. Sci. Technol., 2012, 72(11), p 1291-1297

6. Y. Long, H. Zhang, T. Wang, X. Huang, Y. Li, J. Wu, and H. Chen, High-Strength Ti-6Al-4 V with Ultrafine-Grained Structure Fabricated by High Energy Ball Milling and Spark Plasma Sintering, Mater. Sci. Eng. A, 2013, 585, p 408-414

7. A.M. Soufiani, F. Karimzadeh, and M. Enayati, Formation Mechanism and Characterization of Nanostructured Ti6Al4V Alloy Prepared by Mechanical Alloying, Mater. Des., 2012, 37, p 152-160

8. V.A.R. Henriques, P.P.D. Campos, C.A.A. Cairo, and J.C. Bressiani, Production of Titanium Alloys for Advanced Aerospace Systems by Powder Metallurgy, Mater. Res., 2005, 8(4), p 443-446

9. M.B. Ndaliman, K.C. Bala, A.A. Khan, M.Y. Ali, U. Abdullahi, A.A. Abdulmumin, The Effects of Sliding Parameters on Dry Wear Characteristics of Ti-6Al-4 V Alloy. Adv. Mater. Res. (Trans Tech Publ, 2015), pp. 213-216

10. N.S.M. El-Tayeb, T.C. Yap, and P.V. Brevern, Wear Characteristics of Titanium Alloy Ti54 for Cryogenic Sliding Applications, Tribol. Int., 2010, 43(12), p 2345-2354

11. K.S. Munir, Y. Li, M. Qian, and C. Wen, Identifying and Understanding the Effect of Milling Energy on the Synthesis of Carbon Nanotubes Reinforced Titanium Metal Matrix Composites, Carbon, 2016, 99, p 384-397

12. K.S. Munir, Y. Li, D. Liang, M. Qian, W. Xu, and C. Wen, Effect of Dispersion Method on the Deterioration, Interfacial Interactions and Re-agglomeration of Carbon Nanotubes in Titanium Metal Matrix Composites, Mater. Des., 2015, 88, p 138-148

13. B.I. Yakobson and P. Avouris, Mechanical Properties of Carbon Nanotubes, Springer, Carbon nanotubesed., 2001, p 287-327

14. T.W. Ebbesen, Carbon Nanotubes, Phys. Today, 1996, 49(6), p 26-35

15. M. Dresselhaus, G. Dresselhaus, and R. Saito, Physics of Carbon Nanotubes, Carbon, 1995, 33(7), p 883-891

16. P.J.F. Harris, Carbon Nanotube Composites, Int. Mater. Rev., 2004, 49(1), p 31-43

17. P. Ajayan, Nanotubes from Carbon, Chem. Rev., 1999, 99(7), p 17871800

18. K.S. Munir and C. Wen, Deterioration of the Strong sp2 Carbon Network in Carbon Nanotubes during the Mechanical Dispersion Processing-A Review, Crit. Rev. Solid State Mater. Sci., 2016, 41(5), p 347-366

19. M.S. Dresselhaus, G. Dresselhaus, and P.C. Eklund, Science of Fullerenes and Carbon Nanotubes: Their Properties and Applications, Academic press, Cambridge, 1996

20. K.S. Munir, P. Kingshott, and C. Wen, Carbon Nanotube Reinforced Titanium Metal Matrix Composites Prepared by Powder Metallurgy-A Review, Crit. Rev. Solid State Mater. Sci., 2015, 40(1), p $38-55$

21. S. Bakshi, D. Lahiri, and A. Agarwal, Carbon Nanotube Reinforced Metal Matrix Composites-A Review, Int. Mater. Rev., 2010, 55(1), p 41-64

22. S. Li, B. Sun, H. Imai, and K. Kondoh, Powder Metallurgy Ti-TiC Metal Matrix Composites Prepared by In situ Reactive Processing of Ti-VGCFs System, Carbon, 2013, 61, p 216-228

23. E.T. Thostenson, Z. Ren, and T.-W. Chou, Advances in the Science and Technology of Carbon Nanotubes and Their Composites: A Review, Compos. Sci. Technol., 2001, 61(13), p 1899-1912
24. K. Kondoh, T. Threrujirapapong, H. Imai, J. Umeda, and B. Fugetsu, CNTs/TiC Reinforced Titanium Matrix Nanocomposites via Powder Metallurgy and Its Microstructural and Mechanical Properties, $J$. Nanomater, 2008, 2008, p 76

25. S. Li, B. Sun, H. Imai, T. Mimoto, and K. Kondoh, Powder Metallurgy Titanium Metal Matrix Composites Reinforced with Carbon Nanotubes and Graphite, Compos. A Appl. Sci. Manuf., 2013, 48, p 57-66

26. H. Jia, Z. Zhang, Z. Qi, G. Liu, and X. Bian, Formation of Nanocrystalline TiC from Titanium and Different Carbon Sources by Mechanical Alloying, J. Alloy. Compd., 2009, 472(1), p 97-103

27. A. Esawi, K. Morsi, A. Sayed, M. Taher, and S. Lanka, Effect of Carbon Nanotube (CNT) Content on the Mechanical Properties of CNT-Reinforced Aluminium Composites, Compos. Sci. Technol., 2010, 70(16), p 2237-2241

28. K.S. Munir, D.T. Oldfield, and C. Wen, Role of Process Control Agent in the Synthesis of Multi-Walled Carbon Nanotubes Reinforced Titanium Metal Matrix Powder Mixtures, Adv. Eng. Mater., 2016, 18(2), p 294-303

29. J.Z. Liao, M.J. Tan, R.V. Ramanujan, S. Shukla, Carbon Nanotube Evolution in Aluminum Matrix During Composite Fabrication Process. Mater. Sci. Forum (Trans Tech Publ, 2011), pp. 294-297

30. Y. Kim, T. Hayashi, Y. Fukai, M. Endo, T. Yanagisawa, and M. Dresselhaus, Effect of Ball Milling on Morphology of Cup-Stacked Carbon Nanotubes, Chem. Phys. Lett., 2002, 355(3), p 279-284

31. L. Lü and M.O. Lai, Mechanical Alloying, Springer, Berlin, 2013

32. K.S. Munir, M. Qian, Y. Li, D.T. Oldfield, P. Kingshott, D.M. Zhu, and C. Wen, Quantitative Analyses of MWCNT-Ti Powder Mixtures using Raman Spectroscopy: The Influence of Milling Parameters on Nanostructural Evolution, Adv. Eng. Mater, 2015, 17(11), p 1660-1669

33. M.T. Hassan, A.M. Esawi, and S. Metwalli, Effect of Carbon Nanotube Damage on the Mechanical Properties of Aluminium-Carbon Nanotube Composites, J. Alloy. Compd., 2014, 607, p 215-222

34. P. Gill and N. Munroe, Study of Carbon Nanotubes in $\mathrm{Cu}-\mathrm{Cr}$ Metal Matrix Composites, J. Mater. Eng. Perform., 2012, 21(11), p 2467-2471

35. Y. Oh, J. Choi, Y. Kim, K. Kim, and S. Baik, The Effects of Ball Milling Process on the Diameter Dependent Fracture of Single Walled Carbon Nanotubes, Scripta Mater, 2007, 56(9), p 741-744

36. L. Ci, Z. Ryu, N.Y. Jin-Phillipp, and M. Rühle, Investigation of the Interfacial Reaction Between Multi-walled Carbon Nanotubes and Aluminum, Acta Mater., 2006, 54(20), p 5367-5375

37. T.P. Yadav, R.M. Yadav, and D.P. Singh, Mechanical Milling: A Top Down Approach for the Synthesis of Nanomaterials and Nanocomposites, Nanosci. Nanotechnol., 2012, 2(3), p 22-48

38. A. Nouri and C. Wen, Surfactants in Mechanical Alloying/Milling: A Catch-22 Situation, Crit. Rev. Solid State Mater. Sci., 2014, 39(2), p 81-108

39. C. Machio, H. Chikwanda, and S. Chikosha, Effect of Process Control Agent (PCA) on the Characteristics of Mechanically Alloyed Ti-Mg Powders, J. South Afr. Inst. Min. Metall., 2011, 111(3), p 149-153

40. A. Canakci, T. Varol, and S. Ozsahin, Analysis of the Effect of a New Process Control Agent Technique on the Mechanical Milling Process Using a Neural Network Model: Measurement and Modeling, Measurement, 2013, 46(6), p 1818-1827

41. Z. Xiao, Z. Li, M. Fang, S. Xiong, X. Sheng, and M. Zhou, Effect of Processing of Mechanical Alloying and Powder Metallurgy on Microstructure and Properties of $\mathrm{Cu}-\mathrm{Al}-\mathrm{Ni}-\mathrm{Mn}$ Alloy, Mater. Sci. Eng. A, 2008, 488(1), p 266-272

42. A. Nouri, P. Hodgson, and C. Wen, Effect of Process Control Agent on the Porous Structure and Mechanical Properties of a Biomedical Ti$\mathrm{Sn}-\mathrm{Nb}$ Alloy Produced by Powder Metallurgy, Acta Biomater, 2010, 6(4), p 1630-1639

43. A. Nouri, P.D. Hodgson, and C.E. Wen, Study on the Role of Stearic Acid and Ethylene-bis-stearamide on the Mechanical Alloying of a Biomedical Titanium Based Alloy, Metall. Mater. Trans. A, 2010, 41(6), p 1409-1420

44. A. Nouri, P.D. Hodgson, and C. Wen, Effect of Ball-milling Time on the Structural Characteristics of Biomedical Porous Ti-Sn-Nb Alloy, Mater. Sci. Eng. C, 2011, 31(5), p 921-928

45. C. Suryanarayana, Mechanical Alloying and Milling, Prog. Mater. Sci., 2001, 46(1), p 1-184

46. M. Schoenitz, X. Zhu, and E.L. Dreizin, Carbide Formation in Al-Ti Mechanical Alloys, Scripta Mater., 2005, 53(9), p 1095-1099

47. D. Gavrilov, O. Vinogradov, W. Shaw, Computer Simulation of Mechanical Alloying in a Shaker Ball Mill, in Tenth International 
Conference on Composite Materials. III. Processing and Manufacturing (1995), pp. 11-17

48. F.H. Gojny, J. Nastalczyk, Z. Roslaniec, and K. Schulte, Surface Modified Multi-Walled Carbon Nanotubes in CNT/Epoxy-Composites, Chem. Phys. Lett., 2003, 370(5), p 820-824

49. Y. Feng, H. Zhang, Y. Hou, T.P. McNicholas, D. Yuan, S. Yang, L. Ding, W. Feng, and J. Liu, Room Temperature Purification of Few-Walled Carbon Nanotubes with High Yield, ACS Nano, 2008, 2(8), p 1634-1638

50. O.S. Deepak, M. Jassala, and A.K. Agrawala, Simple and Fast Method for Purifying Single-Walled Carbon Nanotubes, Indian J. Fibre Text. Res., 2009, 34, p 374-376

51. R. Fleischer, D. Dimiduk, and H. Lipsitt, Intermetallic Compounds for Strong High-Temperature Materials: Status and Potential, Annu. Rev. Mater. Sci., 1989, 19(1), p 231-263

52. H. Zhang, C.H. Sun, F. Li, H.X. Li, and H.M. Cheng, Purification of Multiwalled Carbon Nanotubes by Annealing and Extraction Based on the Difference in Van Der Waals Potential, J. Phys. Chem. B, 2006 110(19), p 9477-9481

53. S. Vivekchand, A. Govindaraj, M.M. Seikh, and C. Rao, New Method of Purification of Carbon Nanotubes Based on Hydrogen Treatment, $J$. Phys. Chem. B, 2004, 108(22), p 6935-6937

54. P. Delhaes, M. Couzi, M. Trinquecoste, J. Dentzer, H. Hamidou, and C. Vix-Guterl, A Comparison Between Raman Spectroscopy and Surface
Characterizations of Multiwall Carbon Nanotubes, Carbon, 2006 , 44(14), p 3005-3013

55. V. Datsyuk, M. Kalyva, K. Papagelis, J. Parthenios, D. Tasis, A. Siokou, I. Kallitsis, and C. Galiotis, Chemical Oxidation of Multiwalled Carbon Nanotubes, Carbon, 2008, 46(6), p 833-840

56. J.H. Lehman, M. Terrones, E. Mansfield, K.E. Hurst, and V. Meunier, Evaluating the Characteristics of Multiwall Carbon Nanotubes, Carbon, 2011, 49(8), p 2581-2602

57. Y.A. Kim, T. Hayashi, K. Osawa, M.S. Dresselhaus, and M. Endo, Annealing Effect on Disordered Multi-wall Carbon Nanotubes, Chem. Phys. Lett., 2003, 380(3-4), p 319-324

58. L.L. Ye and M.X. Quan, Synthesis of Nanocrystalline TiC Powders by Mechanical Alloying, Nanostruct. Mater., 1995, 5(1), p 25-31

59. B. Lohse, A. Calka, and D. Wexler, Synthesis of TiC by Controlled Ball Milling of Titanium and Carbon, J. Mater. Sci., 2007, 42(2), p 669-675

60. W. Cai, X. Feng, and J. Sui, Preparation of Multi-walled Carbon Nanotube-reinforced TiNi Matrix Composites from Elemental Powders by Spark Plasma Sintering, Rare Met., 2012, 31(1), p 48-50

61. A.C. Ferrari, Raman Spectroscopy of Graphene and Graphite: Disorder, Electron-Phonon Coupling, Doping and Nonadiabatic Effects, Solid State Commun., 2007, 143(1-2), p 47-57 